\title{
Colonization resistance of the digestive tract in conventional and antibiotic-treated mice
}

\author{
BY D. VAN DER WAAIJ, J. M. BERGHUIS-DE VRIES \\ AND J. E. C. LEKKERKERK-VAN DER WEES \\ Radiobiological Institute TNO, Lange Kleiweg, \\ Rijswijk, The Netherlands \\ (Received 1 March 1971)
}

\begin{abstract}
SUMMARY
The effect of oral administration of antibiotics on the intestinal flora of conventional mice and their resistance to colonization by orally introduced Escherichia coli, Klebsiella pneumoniae and Pseudomonas aeruginosa was studied. Colonization resistance (CR) was expressed as the log of the oral bacterial dose followed by a persistent take in $50 \%$ of the contaminated animals. The intestinal flora was virtually eliminated by the antibiotics and this elimination was accompanied by a precipitous fall of CR. CR gradually returned to normal values during the period of repopulation of the intestinal tract by the organisms surviving the treatment. Antibiotic treatment resulted in the disappearance of Enterobacteriaceae, enterococci, staphylococci and yeasts and, under appropriate housing conditions, the animals remained free of these organisms indefinitely. Germ-free mice contaminated with the intestinal flora of an antibiotic-treated animal and their offspring housed in a germ-free isolator showed high values of CR. Their intestinal flora consisted of anaerobic bacteria only. Apparently, these anaerobes are responsible for $\mathrm{CR}$ in these and in conventional mice.
\end{abstract}

\section{INTRODUCTION}

In a previous paper the selective elimination of Enterobacteriaceae from the digestive tract of mice by administration of an antibiotic mixture was reported (van der Waaij, 1968). Provided precautions were taken to prevent gross contamination, the animals remained free of Enterobacteriaceae species. Even when placed in conventional animal rooms, it took weeks before the animals became recolonized and they were, apparently, resistant to colonization of their intestinal tract by small numbers of ingested Enterobacteriaceae. We propose to call this resistance colonization resistance (CR).

When it was found that elimination of organisms depends on numbers of orally administered cells, CR could be quantified. In the present investigation, CR was studied quantitatively before, during and after antibiotic treatment. As indicated in Fig. 1 , the period before antibiotic treatment is called phase $I$, the actual treatment period phase II and the period in which organisms surviving the treatment repopulate the intestinal tract phase III; phase IV begins when repopulation is completed. The results showed CR to be equal in animals of phases I and IV and 
to be severely decreased in phases II and III. This suggested that CR might be due to organisms present in phase I and surviving the antibiotic treatment. In order to analyse the mechanism of $\mathrm{CR}$, germ-free mice were contaminated with the flora of one phase IV mouse. These animals and their offspring were found to be highly resistant to colonization. Apparently, their intestinal flora induces CR and it is supposed to produce a hypothetical factor, colonization resistance factor (CRF). For convenience, these mice are called CRF-mice. Their intestinal flora, which consisted of anaerobic bacteria only, was subjected to a detailed study to be reported separately (Wensinck \& Ruseler-van Embden, 1971).

\section{MATERIALS AND METHODS}

Mice

Conventional females from a non-inbred Pseudomonas aeruginosa-free Swiss mouse stock (ND 2) (van der Waaij, Zimmerman \& van Bekkum, 1963), varying in age between 9 and 12 weeks and weighing between 25 and $35 \mathrm{~g}$., were used. In a few experiments CRF-mice, the offspring of germ-free (GF) mice contaminated with the flora of one phase IV mouse (see Introduction), were used. These animals were maintained under GF conditions and were of the same stock and age and weight range as the conventional animals.

\section{Antibiotic sensitivity of faecal organisms}

Applying the sensitivity test as used in the selective decontamination of monkeys (van der Waaij, de Vries \& Lekkerkerk, 1970) the combination of streptomycin and neomycin was found to be optimal; therefore these antibiotics were used in the present experiments. The mixture was supplied in a concentration of $5 \mathrm{mg}$. of each antibiotic per ml. of drinking water to which pimaricin was also added (100 $\mu \mathrm{g} . / \mathrm{ml}$.$) .$

\section{Housing and isolation conditions}

These will be described for each of the four phases of the selective decontamination procedure. Phase I: animals were housed singly on wire mesh $1 \mathrm{~cm}$. above three sheets of filter paper in autoclaved polycarbonate cages and supplied with autoclaved pellets and drinking water acidified to $\mathrm{pH} 3$ after being autoclaved. Cages were placed on racks closed with plastic sheets (van der Waaij \& Sturm, 1968). Phase II: the cages were transferred to peracetic acid-sterilized laminar cross-flow benches and placed at a distance of $10 \mathrm{~cm}$. one from another; the autoclaved drinking water to which antibiotics were added was not acidified. Phase III: the same conditions as phase II but antibiotics were not supplied and drinking water was acidified. During manipulations inside the cabinet long peracetic acidsterilized neoprene gloves were worn. The phase II and III procedures have been described in detail before (van der Waaij \& Andreas, 1971). Phase IV: the same conditions as phase $I$.

The offspring of GF mice contaminated with the faecal flora of one phase IV mouse (CRF-mice) were housed in a GF plastic isolator. When used for quantitative 
study of CR the animals were removed from the isolator and housed as the animals of phase III.

\section{Evaluation of $C R$}

Overnight cultures of mouse-derived streptomycin- and neomycin-resistant strains of Escherichia coli, Klebsiella pneumoniae and Pseudomonas aeruginosa in brain-heart infusion (Difco) were tenfold serially diluted. The phase I and IV mice received $10^{9}, 10^{7}$ and $10^{5}$ cells per dose of $0.1 \mathrm{ml}$, the phase II mice $10^{3}$ and $10^{2}$ and the phase III mice $10^{8}, 10^{6}$ and $10^{4}$ cells. The bacteria were administered to the mice by introducing the cone of a $2 \mathrm{ml}$. injection syringe into the mouth and slowly delivering $0.1 \mathrm{ml}$. of the suspensions. Each challenge dose was given to 20 mice and different mice were challenged in the four phases. Faeces were cultured at intervals for the presence of the organisms introduced.

\section{Faeces cultures}

Fresh faeces of phase III and IV mice were cultured weekly in brain-heart infusion; subcultures were made on brain-heart infusion agar, Endo agar, staphylococcus medium no. 110 and aesculin-azide agar which was prepared according to Sneath (1956). Faeces of animals contaminated with streptomycin- and neomycin-resistant strains of E. coli, Ps. aeruginosa or Kl. pneumoniae to evaluate CR were cultured 2-3 times a week in brain-heart infusion containing streptomycin (10 mg. $/ \mathrm{ml}$.) and bacitracin ( $1 \mathrm{mg} . / \mathrm{ml}$.); subcultures were made on Endo agar. The endogenous Gram-negative flora of phase I mice was sensitive to $100 \mu \mathrm{g} . / \mathrm{ml}$. of streptomycin.

\section{RESULTS}

\section{Effect of the phase II antibiotic treatment on the intestinal flora}

More than 200 conventional mice were treated and all became free of Enterobacteriaceae, enterococci, staphylococci, yeasts and fungi. They were free of $P s$. aeruginosa when entering phase I.

\section{$C R$ in phases $I-I V$}

The results in Fig. 2 show that the number of phase I and IV animals excreting the three contaminants gradually decreases and that this decrease is more rapid as the dose is lower. In contrast, persistent colonization with the three organisms takes place in all phase II animals.

In order better to compare the CR of mice to different organisms, CR was expressed as the log of the oral dose of an organism resulting in a colonization in $50 \%$ of the animals for at least 2 weeks. Thus, the CR of phase I mice to $E$. coli was 7 , that of phase II mice to Ps. aeruginosa $<2$ and that of phase IV mice to $K l$. pneumoniae $>9$. From a comparison of $\mathrm{CR}$ in phases II and IV it is clear that CR increases to normal values during phase III. Recovery in this phase is shown in Fig. 1 in which CR was measured with $E$. coli. 


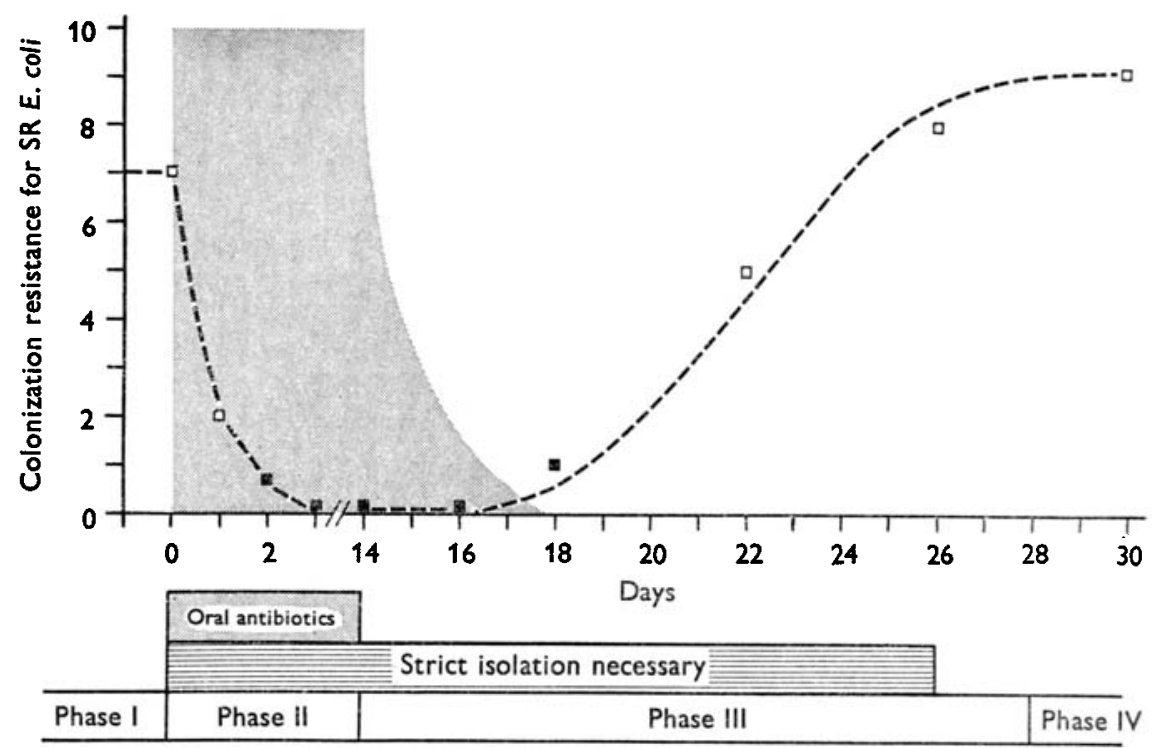

Fig. 1. Colonization resistance of mice to Escherichia coli in phases I-IV. Twenty mice per group. $\square$, CR $\geqslant 2 ; \boldsymbol{D}, \mathrm{CR}<2$.

Phase I
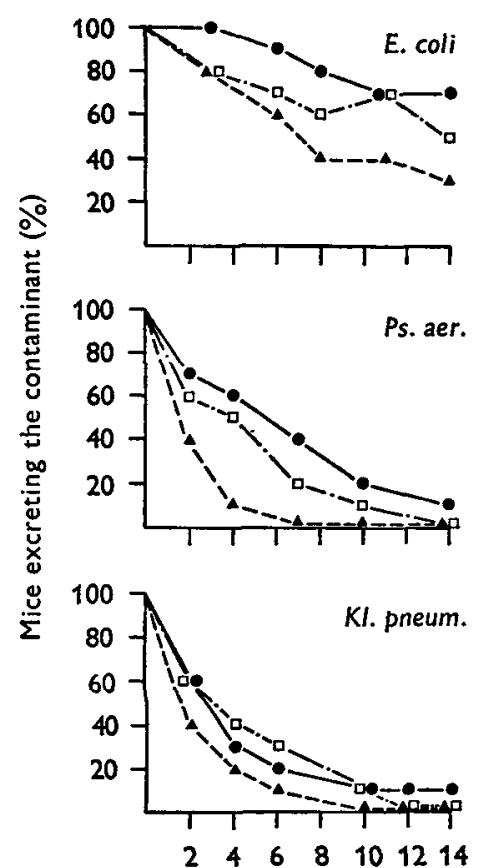

Phase II
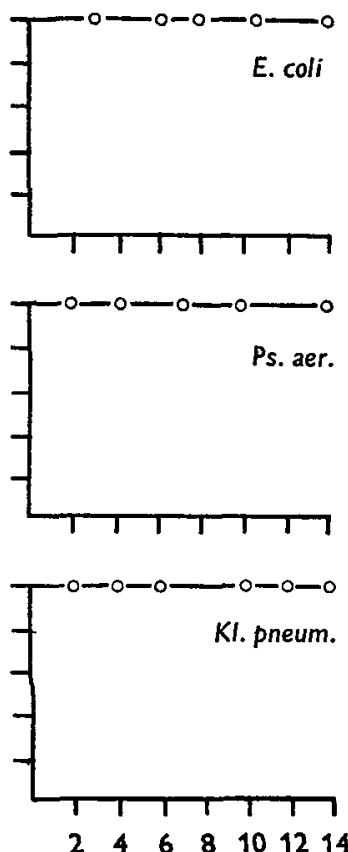

Days after contamination

Phase IV

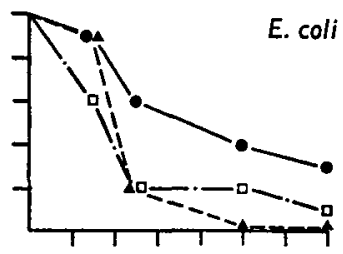

Ps. aer.
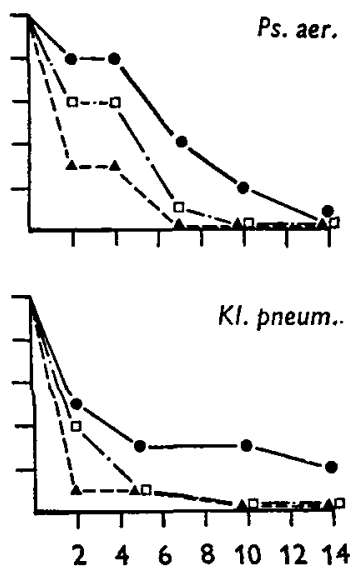

Fig. 2. Percentage of mice excreting Escherichia coli, Pseudomonas aeruginosa and Klebsiella pneumoniae at different intervals after oral contamination in phase I, II and IV. Oral dose: $O, 10^{9} ; \square, 10^{7} ; \Delta, 10^{5} ; 0,10^{2}$. Mice were contaminated 2 days after the onset of antibiotic treatment. 


\section{$C R$ in $C R F$-mice}

As shown in Fig. 3, the pattern of elimination of $E$. coli by CRF-mice closely resembles elimination by phase IV mice, their CR to this organism being about 9 . Taking into account that the flora of CRF-mice came from one phase IV mouse and that the flora of this animal represents the fraction of the conventional intestinal flora which survived antibiotic treatment, the results with CRF-mice suggest that their flora consists of organisms responsible for CR in conventional mice.

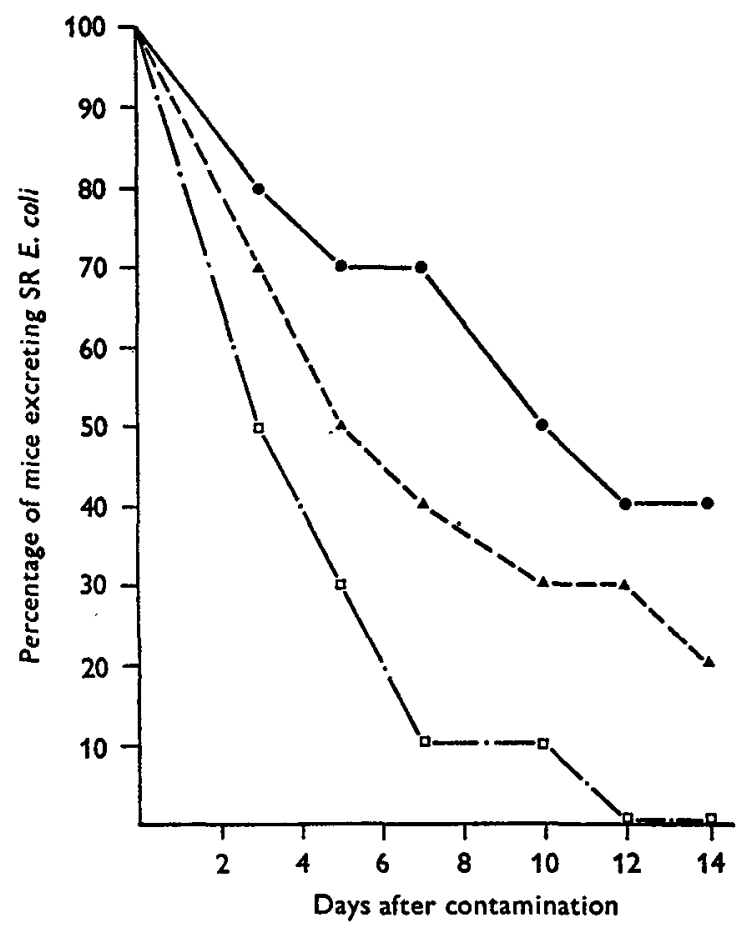

Fig. 3. Percentage of CRF-mice excreting Escherichia coli following three different oral doses. Oral dose: $O, 10^{9} ; \Delta, 10^{7} ; \square, 10^{5}$.

\section{DISCUSSION}

The term 'colonization resistance' (CR) seems appropriate for the process leading to elimination of orally introduced organisms. By expressing CR as the $\log$ of the oral dose of bacteria which produces a long-lasting take in $50 \%$ of the contaminated animals elimination of different organisms can be quantitatively compared. Values of $\mathrm{CR}$ in conventional mice were found to be slightly different for the strains of $E$. coli, $K l$. pneumoniae and $P$ s. aeruginosa tested. Immediately after the onset of oral administration of antibiotics CR decreases and the values remain very low during the period of antibiotic treatment. Decrease and subsequent recovery of $\mathrm{CR}$ in the post-treatment phase coincide with profound changes of the intestinal flora. The antibiotics virtually eliminate this flora and the intestinal tract is gradually repopulated by organisms surviving the treatment. Comparable 
results were obtained when the effect of antibiotic treatment on susceptibility to infection with pathogenic organisms was studied. Single oral doses of streptomycin were followed within $24 \mathrm{hr}$. by a striking increase of susceptibility to Salmonella enteritidis (Bohnhoff \& Miller, 1962). Susceptibility gradually decreased to that of untreated mice and decrease was even more rapid when normal mice were housed together with streptomycin-treated animals. Furthermore, though coliform bacilli multiplied extensively and persisted in all parts of the gastro-intestinal tract of germ-free mice, their numbers fell precipitously when these mice were fed the intestinal contents of healthy pathogen-free mice (Schaedler, Dubos \& Costello, 1965). These data clearly demonstrate the important role of the resident intestinal flora in host resistance to orally introduced organisms.

So far, no definite conclusions have been drawn about the intestinal residents responsible for $\mathrm{CR}$ and the mechanism by which resistance is brought about. Our results suggest that $\mathrm{CR}$ is due to those anaerobes in the conventional intestine which survive the antibiotic treatment and subsequently repopulate the intestinal tract. Studies of the caecal flora of the offspring of germ-free mice contaminated with these anaerobes (CRF-mice) show that five species are represented. Lactobacilli and Bacteroides, the predominant anaerobes of conventional mice, are absent and the major constituents are tapered rods belonging to the genus Clostridium (Wensinck \& Ruseler-van Embden, 1971). The inability of lactobacilli and Bacteroides to reduce coli populations in gnotobiotic mice (Schaedler et al. 1965) supports the view that in conventional animals $\mathrm{CR}$ is also induced by one or more of the five anaerobes present in CRF-mice. Results from preliminary experiments with germ-free mice contaminated with various combinations of these species strongly suggest that $\mathrm{CR}$ is due to the presence of a large, Gram-labile tapered Clostridium.

The elimination of orally introduced organisms has been ascribed to mechanical and chemical factors. The normal flora has been shown to accelerate the propulsion of gastro-intestinal contents (Abrams \& Bishop, 1966, 1967), to reduce the volume of the caecum characteristically enlarged in germ-free and antibiotic-treated animals (Savage \& Dubos, 1968; van der Waaij, 1969) and to produce volatile acids which are thought to inhibit the multiplication of Salmonella in the large bowel (Meynell, 1963; Bohnhoff, Miller \& Martin, 1964a, $b$ ). In this respect it is important to note that the caecum volume of CRF-mice is normal and that the anaerobes isolated produce several volatile acids in vitro (Wensinck \& Ruseler-van Embden, 1971). It is unlikely that the immune system contributes to $\mathrm{CR}$ : both the high values in CRF-mice, born and raised in the germ-free isolator, and the immediate drop of $\mathrm{CR}$ in conventional animals after the onset of antibiotic treatment, are difficult to explain when CR is supposed to be antibody-dependent.

\section{REFERENCES}

Agrams, G. D. \& Bishop, J. E. (1966). Effect of the normal microbial flora on the resistance of the small intestine to infection. Journal of Bacteriology 92, 1604.

Abrams, G. D. \& BisHop, J. E. (1967). Effect of the normal microbial flora on gastro-intestinal motility. Proceedings of the Society for Experimental Biology and Medicine 126, 301.

BoHNhoFf, M. \& Miller, C. P. (1962). Enhanced susceptibility to Salmonella infection in streptomycin-treated mice. Journal of Infectious Diseases 111, 117. 
Bohnhoff, M., Muller, C. P. \& Martin, W. R. (1964a). Resistance of the mouse's intestinal tract to experimental Salmonella infection. I. Factors which interfere with the initiation of infection by oral inoculation. Journal of Experimental Medicine 120, 805.

Bonnhoff, M., Mrller, C. P. \& Martin, W. R. (1964b). Resistance of the mouse's intestinal tract to experimental Salmonella infection. II. Factors responsible for its loss following streptomycin treatment. Journal of Experimental Medicine 120, 817.

MeynelL, G. G. (1963). Antibacterial mechanisms of the mouse gut. II. The role of Eh and volatile fatty acids in the normal gut. British Journal of Experimental Pathology 44, 209.

Savage, D. C. \& Dubos, R. (1968). Alterations in the mouse cecum and its flora produced by antibacterial drugs. Journal of Experimental Medicine 128, 97.

Schaedler, R. W., Dubos, R. \& Costello, R. (1965). Association of germfree mice with bacteria isolated from normal mice. Journal of Experimental Medicine 122, 77.

Sneath, P. H. A. (1956). Cultural and biochemical characteristics of the genus Chromobacterium. Journal of General Microbiology 15, 70.

WAatJ, D. VAN DER (1968). The persistent absence of Enterobacteriaceae from the intestinal flora of mice following antibiotic treatment. Journal of Infectious Diseases $118,32$.

WAAIJ, D. VAN DER (1969). Similarities between GF mice and mice with an antibiotic decontaminated digestive tract. In Germfree Biology, p. 181. New York: Plenum Press.

WaAiJ, D. van der \& Andreas, A. H. (1971). Prevention of airborne contamination and cross-contamination in germ-free mice by laminar flow. Journal of Hygiene 69, 83.

WAaIJ, D. van DER \& Sturm, C. A. (1968). Antibiotic decontamination of the digestive tract of mice. Technical procedures. Laboratory Animal Care 18, 1.

WAatJ, D. vaN Der, VRIES, J. M. DE \& LEkkERKERK, J. E. C. (1970). Eliminating bacteria from monkeys with antibiotics. In Infections and Immunosuppression in Subhuman Primates, p. 21. Copenhagen: Munksgaard.

WaAi, D. van der, Zimmerman, W. M. T. \& Bekkum, D. W. van (1963). An outbreak of Pseudomonas aeruginosa infection in a colony previously free of this infection. Laboratory Animal Care 13, 46.

Wensinck, F. \& Ruseler-van Embden, J. G. H. (1971). The intestinal flora of colonization resistant mice. Journal of Hygiene 69, 413. 